\title{
Quasistationarity in a model of classical spins with long-range interactions
}

\author{
Shamik Gupta and David Mukamel \\ Physics of Complex Systems, Weizmann Institute of Science, Rehovot 76100, Israel \\ E-mail: shamik.gupta@weizmann.ac.il, david.mukamel@weizmann.ac.il
}

\begin{abstract}
Systems with long-range interactions, while relaxing towards equilibrium, sometimes get trapped in long-lived non-Boltzmann quasistationary states (QSS) which have lifetimes that grow algebraically with the system size. Such states have been observed in models of globally coupled particles that move under Hamiltonian dynamics either on a unit circle or on a unit spherical surface. Here, we address the ubiquity of QSS in longrange systems by considering a different dynamical setting. Thus, we consider an anisotropic Heisenberg model consisting of classical Heisenberg spins with mean-field interactions and evolving under classical spin dynamics. Our analysis of the corresponding Vlasov equation for time evolution of the phase space distribution shows that in a certain energy interval, relaxation of a class of initial states occurs over a timescale which grows algebraically with the system size. We support these findings by extensive numerical simulations. This work further supports the generality of occurrence of QSS in long-range systems evolving under Hamiltonian dynamics.

PACS numbers: 05.20.-y, 05.70.Ln, 05.40.-a
\end{abstract}




\section{Introduction}

Long-range interacting systems have attracted considerable interest in recent years [1 6]. These systems are characterized by an interparticle potential which in $d$ dimensions decays at large separation, $r$, as $1 / r^{\alpha}$, with $\alpha \leq d$. Examples include non-neutral plasmas [7], dipolar ferroelectrics and ferromagnets [8], self-gravitating systems [9], two-dimensional geophysical vortices [10], etc. Long-range interactions lead to non-additivity, whereby thermodynamic quantities scale superlinearly with the system size. This may result in equilibrium properties which are unusual for short-range systems, e.g., a negative microcanonical specific heat [11,12], inequivalence of statistical ensembles [13, 14], and many others [15].

As for the dynamical properties, long-range systems often exhibit broken ergodicity [14, 16] and intriguingly slow relaxation towards equilibrium [10, 14, 17, 20]. Such slow relaxation has been discussed in the context of self-gravitating systems (see, e.g., [21,22]), and has recently been demonstrated in a model of globally coupled particles (inertial rotors) moving on a unit circle and evolving under deterministic Hamilton dynamics. In this socalled Hamiltonian mean-field (HMF) model, it has been found that for a wide class of initial distributions, the relaxation time diverges with the system size [17]. For some energy interval, the relaxation proceeds through intermediate long-lived quasistationary states (QSS). These non-Boltzmann states exhibit slow relaxation of thermodynamic observables, and have a lifetime which grows algebraically with the system size [18, 23]. As a result, the system in the thermodynamic limit never attains the Boltzmann-Gibbs equilibrium but remains trapped in the QSS. At other energies, however, relaxation occurs faster on a time which grows logarithmically with the system size. Quasistationary states in the HMF model exhibit interesting features like anomalous diffusion and non-Gaussian velocity distributions [24 26]. Generalization of the model to include anisotropy terms in the energy [23], and to particles which are confined to move on a spherical surface rather than on a circle [27], have also shown the existence of QSS. All these models evolve under deterministic Hamiltonian dynamics. The robustness of quasistationarity to stochastic dynamical processes has also been analyzed, where it is found that QSS exist only as a crossover phenomenon. Under such dynamics, these states have a finite relaxation time which is determined by the rate of the stochastic process [28 33].

In this work, we address the issue of ubiquity of QSS in long-range systems by examining a different dynamical model for its existence than the HMF model. To this end, we consider an anisotropic Heisenberg model with mean-field interactions. The model comprises globally coupled three-component Heisenberg spins evolving under classical spin dynamics. The dynamics is thus very different from particle dynamics of previously studied long-range systems that have shown the existence of QSS.

Our model has an equilibrium phase diagram with a continuous transition from a low-

energy magnetic phase to a high-energy non-magnetic phase. Analysis of the Vlasov equation 
for evolution of the phase space distribution shows that, as in previously studied models, our model exhibits relaxation over times that grow either logarithmically or algebraically with the system size. At low energies, a non-magnetic initial state is dynamically unstable. It relaxes to the stable magnetically-ordered state on a logarithmic timescale. At higher energies, but within the magnetic phase, the non-magnetic state becomes linearly stable, and its relaxation to equilibrium occurs on an algebraically growing timescale so that QSS are observed. These results, obtained by analyzing the marginal stability of the Vlasov equation, are supported by extensive numerical simulations. This analysis yields further evidence for the occurrence of QSS in generic long-range systems.

\section{The model}

We start by defining the model of study. It consists of $N$ globally coupled classical Heisenberg spins of unit length, $\mathbf{S}_{i}=\left(S_{i x}, S_{i y}, S_{i z}\right), i=1,2, \ldots, N$. In terms of spherical polar angles $\theta_{i} \in[0, \pi]$ and $\phi_{i} \in[0,2 \pi]$ for the orientation of the $i$-th spin, one has $S_{i x}=\sin \theta_{i} \cos \phi_{i}, S_{i y}=\sin \theta_{i} \sin \phi_{i}, S_{i z}=\cos \theta_{i}$. The Hamiltonian of the model is given by

$$
H=-\frac{J}{2 N} \sum_{i, j=1}^{N} \mathbf{S}_{i} \cdot \mathbf{S}_{j}+D \sum_{i=1}^{N} S_{i z}^{2},
$$

where the first term with $J>0$ describes a ferromagnetic mean-field like coupling, while the last term gives the energy due to a local anisotropy. We take $D>0$ such that at

equilibrium, the energy is lowered by having the magnetization $\mathbf{m}=(1 / N) \sum_{i=1}^{N} \mathbf{S}_{i}$ pointing in the $x y$ plane. The coupling constant $J$ is scaled by $1 / N$ to make the energy extensive, in accordance with the Kac prescription 34. However, the system continues to remain nonadditive in the sense that it cannot be trivially divided into independent macroscopic parts, as can be achieved with short-range systems. In this work, we take $J=1$ and the Boltzmann constant $k_{B}=1$.

The time evolution of the model (1) is governed by the set of first-order differential equations

$$
\frac{d \mathbf{S}_{i}}{d t}=\left\{\mathbf{S}_{i}, H\right\} ; \quad i=1,2, \ldots, N
$$

Here the Poisson bracket $\{A, B\}$ for two functions of the spins is obtained by noting that suitable canonical variables for a classical spin are $\phi$ and $S_{z}$, so that in our model, $\{A, B\} \equiv \sum_{i=1}^{N}\left(\partial A / \partial \phi_{i} \partial B / \partial S_{i z}-\partial A / \partial S_{i z} \partial B / \partial \phi_{i}\right)$. It may be rewritten as [35]

$$
\{A, B\}=\sum_{i=1}^{N} \mathbf{S}_{i} \cdot \frac{\partial A}{\partial \mathbf{S}_{i}} \times \frac{\partial B}{\partial \mathbf{S}_{i}} .
$$

Using the above relation, we obtain the equations of motion of the model: 


$$
\begin{aligned}
& \dot{S}_{i x}=S_{i y} m_{z}-S_{i z} m_{y}-2 D S_{i y} S_{i z}, \\
& \dot{S}_{i y}=S_{i z} m_{x}-S_{i x} m_{z}+2 D S_{i x} S_{i z}, \\
& \dot{S}_{i z}=S_{i x} m_{y}-S_{i y} m_{x} .
\end{aligned}
$$

Note that the above equations of motion may also be obtained by considering the corresponding quantum equations and taking the limit of infinite spins.

From Eq. ([6), one finds by summing over $i$, that $m_{z}$ is a constant of motion. The motion also conserves the total energy and the length of each spin. From Eqs. (4), (5), and (6), the time evolution of the variables $\theta_{i}$ and $\phi_{i}$ are obtained as

$$
\begin{aligned}
& \dot{\theta}_{i}=m_{x} \sin \phi_{i}-m_{y} \cos \phi_{i}, \\
& \dot{\phi}_{i}=m_{x} \cot \theta_{i} \cos \phi_{i}+m_{y} \cot \theta_{i} \sin \phi_{i}-m_{z}+2 D \cos \theta_{i} .
\end{aligned}
$$

Note that the Hamiltonian of the previously studied models of particles moving either on a unit circle [17] or on a spherical surface [27] may also be expressed in terms of spin variables. However, the dynamics of these models is rather different from that of the model considered here. Unlike the present model, all Poisson brackets of the spin variables of these models are set to zero, and the dynamics is generated by a kinetic energy term which is explicitly included in the Hamiltonians and which is absent in the present model. It is worthwhile to point out that even in the large $D$ limit, when the $z$-component of the spins becomes vanishingly small, the dynamics is different from the HMF model with particles moving on a unit circle.

\section{Equilibrium phase diagram}

We now discuss the equilibrium phase diagram of our model. The canonical partition function $Z$ is given by

$$
Z=\int \prod_{i} \sin \theta_{i} d \theta_{i} d \phi_{i} \exp \left[\frac{\beta N m^{2}}{2}-\beta D \sum_{i} \cos ^{2} \theta_{i}\right],
$$

where $\beta$ is the inverse temperature, and $m=\left(m_{x}^{2}+m_{y}^{2}+m_{z}^{2}\right)^{1 / 2}$. Since Eq. (11) describes a mean-field system, it is straightforward to write down expressions for equilibrium quantities like the average magnetization or the average energy. As mentioned above, for $D>0$, the system orders in the $x y$ plane. Choosing the ordering direction as $x$ without loss of generality, the average equilibrium magnetization along $x$, given by $\left\langle m_{x}\right\rangle=\langle\sin \theta \cos \phi\rangle$, is

$$
\left\langle m_{x}\right\rangle=\frac{\int \sin ^{2} \theta \cos \phi d \theta d \phi e^{\beta\left\langle m_{x}\right\rangle \sin \theta \cos \phi-\beta D \cos ^{2} \theta}}{\int \sin \theta d \theta d \phi e^{\beta\left\langle m_{x}\right\rangle \sin \theta \cos \phi-\beta D \cos ^{2} \theta}} .
$$


Close to the critical point, one may expand the above transcendental equation to leading order in $\left\langle m_{x}\right\rangle$ to get

$$
\left\langle m_{x}\right\rangle\left(\int \sin \theta d \theta d \phi e^{-\beta_{c} D \cos ^{2} \theta}-\beta_{c} \int \sin ^{3} \theta \cos ^{2} \phi d \theta d \phi e^{-\beta_{c} D \cos ^{2} \theta}\right)=0 .
$$

The transition temperature $\beta_{c}$ is obtained by equating the bracketed expression to zero, and may be seen to satisfy

$$
\frac{2}{\beta_{c}}=1-\frac{1}{2 \beta_{c} D}+\frac{e^{-\beta_{c} D}}{\sqrt{\pi \beta_{c} D} \operatorname{Erf}\left[\sqrt{\beta_{c} D}\right]} .
$$

Here, $\operatorname{Erf}[x]=\frac{2}{\sqrt{\pi}} \int_{0}^{x} e^{-t^{2}} d t$ is the error function. The critical energy density, $\epsilon_{c}=D\left\langle\cos ^{2} \theta\right\rangle$, is

$$
\epsilon_{c}=D\left(1-\frac{2}{\beta_{c}}\right),
$$

see Fig. (11). In model (11), where the phase transition is continuous, the canonical and microcanonical ensembles are expected to be equivalent [15, 36], and Eq. (13) therefore also yields the microcanonical energy at the transition.

\section{Relaxation towards equilibrium}

To study the dynamical behavior of magnetization, we now analyze the Vlasov equation for evolution of the phase space density [7]. For any mean-field model like ours, this equation faithfully describes the $N$-particle dynamics for finite time and in the limit $N \rightarrow \infty[3,37]$. For the model (1), we study the dynamics by examining a single particle, which is moving in the two-dimensional phase space of its canonical coordinates, $\phi$ and $S_{z}=\cos \theta$, due to the mean-field produced by all other particles. Here, and in the following, the particle index is suppressed for brevity. Let $g(\cos \theta, \phi, t)$ be the probability density in this single-particle phase space, such that $g(\cos \theta, \phi, t) d \cos \theta d \phi$ gives the probability to find the particle with the $z$-component of its spin between $\cos \theta$ and $\cos \theta+d \cos \theta$ and the azimuthal angle between $\phi$ and $\phi+d \phi$ at time $t$. In terms of the canonical coordinates, $\phi$ and $\cos \theta$, the flow in the phase space is divergence free. Conservation of probability then implies vanishing of the total time derivative of $g$, that is,

$$
\frac{d g}{d t}=\frac{\partial g}{\partial t}+\cos \theta \frac{\partial g}{\partial \cos \theta}+\dot{\phi} \frac{\partial g}{\partial \phi}=0,
$$

where dot represents derivative with respect to time. More conveniently, we define a new function, $f(\theta, \phi, t) \equiv g(\cos \theta, \phi, t)$, such that $f(\theta, \phi, t) \sin \theta d \theta d \phi$ gives the probability to find the particle with its angles between $\theta$ and $\theta+d \theta$ and between $\phi$ and $\phi+d \phi$ at time $t$. The equation for the evolution of $f$ is straightforwardly obtained from that of $g$ to yield 
$\partial f / \partial t+\dot{\theta}(\partial f / \partial \theta)+\dot{\phi}(\partial f / \partial \phi)=0$. Using Eqs. (7) and (8) to substitute for $\dot{\theta}$ and $\dot{\phi}$ gives the Vlasov equation for time evolution of $f(\theta, \phi, t)$ as

$$
\begin{aligned}
\frac{\partial f}{\partial t} & =\left[m_{y} \cos \phi-m_{x} \sin \phi\right] \frac{\partial f}{\partial \theta} \\
& -\left[m_{x} \cot \theta \cos \phi+m_{y} \cot \theta \sin \phi-m_{z}+2 D \cos \theta\right] \frac{\partial f}{\partial \phi} .
\end{aligned}
$$

In the above equation, the magnetization components are given by $\left(m_{x}, m_{y}, m_{z}\right)=$ $\int\left(\sin \theta^{\prime} \cos \phi^{\prime}, \sin \theta^{\prime} \sin \phi^{\prime}, \cos \theta^{\prime}\right) f\left(\theta^{\prime}, \phi^{\prime}, t\right) \sin \theta^{\prime} d \theta^{\prime} d \phi^{\prime}$.

Now, consider an initial state prepared by sampling independently for each of the $N$ spins the angle $\phi$ uniformly over $[0,2 \pi]$ and the angle $\theta$ uniformly over an arbitrary interval symmetric about $\pi / 2$. Such a state will have the distribution

$$
f(\theta, \phi, 0)=\frac{1}{2 \pi} p(\theta)
$$

with $p(\theta)$, the distribution for $\theta$, given by

$$
p(\theta)= \begin{cases}\frac{1}{2 \sin a} & \text { if } \theta \in\left[\frac{\pi}{2}-a, \frac{\pi}{2}+a\right], \\ 0 & \text { otherwise. }\end{cases}
$$

We call such an initial state a waterbag state, in analogy with a similar form of an initial state studied in the context of the HMF model. It is easily verified that this non-magnetic state has the energy

$$
\epsilon=\frac{D}{3} \sin ^{2} a
$$

and that the state is stationary under the Vlasov dynamics (15). Let us proceed to analyze the dynamical stability of such a state. Such stability analysis in the context of the HMF model was pursued in [18]. Here, we closely follow the treatment adopted in [23]. We consider finite but large system size $N$, and linearize the Vlasov equation (15) with respect to finite-size fluctuations $\delta f(\theta, \phi, t)$ by expanding $f(\theta, \phi, t)$ as

$$
f(\theta, \phi, t)=\frac{1}{2 \pi} p(\theta)+\lambda \delta f(\theta, \phi, t) .
$$

Since the initial angles of the $N$ spins are sampled independently, the small parameter $\lambda$ is of order $1 / \sqrt{N}$. After linearization, Eq. (15) yields the following equation for $\delta f(\theta, \phi, t)$ :

$$
\frac{\partial \delta f}{\partial t}=\left[m_{y} \cos \phi-m_{x} \sin \phi\right] \frac{1}{2 \pi} \frac{d p(\theta)}{d \theta}-2 D \cos \theta \frac{\partial \delta f}{\partial \phi}
$$

where now $m_{x}$ and $m_{y}$ are linear in $\delta f$.

To study the linear dynamics, we note that at long times, it is dominated by the mode corresponding to the largest eigenfrequency $\omega$ of the linearized equation (20). Since the 
perturbation $\delta f(\theta, \phi, t)$ is $2 \pi$-periodic in $\phi$, one has the following expansion in terms of the Fourier modes $g_{k}(\theta, \omega)$, which is valid after a short initial transient (See [7], Chapter 6):

$$
\delta f(\theta, \phi, t)=\sum_{k} g_{k}(\theta, \omega) e^{i(k \phi+\omega t)} .
$$

The magnetization components are given by

$$
\begin{aligned}
& m_{x}=\pi \int \sin ^{2} \theta^{\prime} d \theta^{\prime}\left(g_{-1}\left(\theta^{\prime}, \omega\right)+g_{1}\left(\theta^{\prime}, \omega\right)\right) e^{i \omega t}, \\
& m_{y}=-i \pi \int \sin ^{2} \theta^{\prime} d \theta^{\prime}\left(g_{-1}\left(\theta^{\prime}, \omega\right)-g_{1}\left(\theta^{\prime}, \omega\right)\right) e^{i \omega t} .
\end{aligned}
$$

It thus follows that the relevant eigenmodes of Eq. (20) have $k= \pm 1$. Using Eq. (21), and the above expressions for $m_{x}$ and $m_{y}$ in Eq. (201), we find that the coefficients $g_{ \pm 1}(\theta, \omega)$ satisfy

$$
g_{ \pm 1}=\frac{d p(\theta)}{d \theta} \frac{1}{2(2 D \cos \theta \pm \omega)} \int_{\pi / 2-a}^{\pi / 2+a} g_{ \pm 1}\left(\theta^{\prime}, \omega\right) \sin ^{2} \theta^{\prime} d \theta^{\prime} .
$$

On multiplying both sides of the above equation by $\sin ^{2} \theta$ and then integrating over $\theta$, we get

$$
I_{ \pm}\left(1-K_{ \pm}\right)=0
$$

where

$$
I_{ \pm}=\int_{\pi / 2-a}^{\pi / 2+a} g_{ \pm 1}(\theta, \omega) \sin ^{2} \theta d \theta
$$

and

$$
K_{ \pm}=\int_{\pi / 2-a}^{\pi / 2+a} \frac{d p(\theta)}{d \theta} \frac{\sin ^{2} \theta}{2(2 D \cos \theta \pm \omega)} d \theta
$$

Since $I_{ \pm} \neq 0$, it then follows from Eq. (25) that the frequency $\omega$ is given by the condition

$$
K_{ \pm}=1 \text {. }
$$

From Eq. (17), we get

$$
\frac{d p(\theta)}{d \theta}=\frac{1}{2 \sin a}\left[\delta\left(\theta-\frac{\pi}{2}+a\right)-\delta\left(\frac{\pi}{2}+a-\theta\right)\right],
$$

which, together with Eqs. (27) and (28), give the following expression for the largest eigenfrequency $\omega$ :

$$
\omega^{2}=4 D^{2} \sin ^{2} a-D \cos ^{2} a .
$$

Expressing the parameter $a$ in the above equation in terms of the energy $\epsilon$ in Eq. (18) finally yields

$$
\omega^{2}=\epsilon(3+12 D)-D .
$$




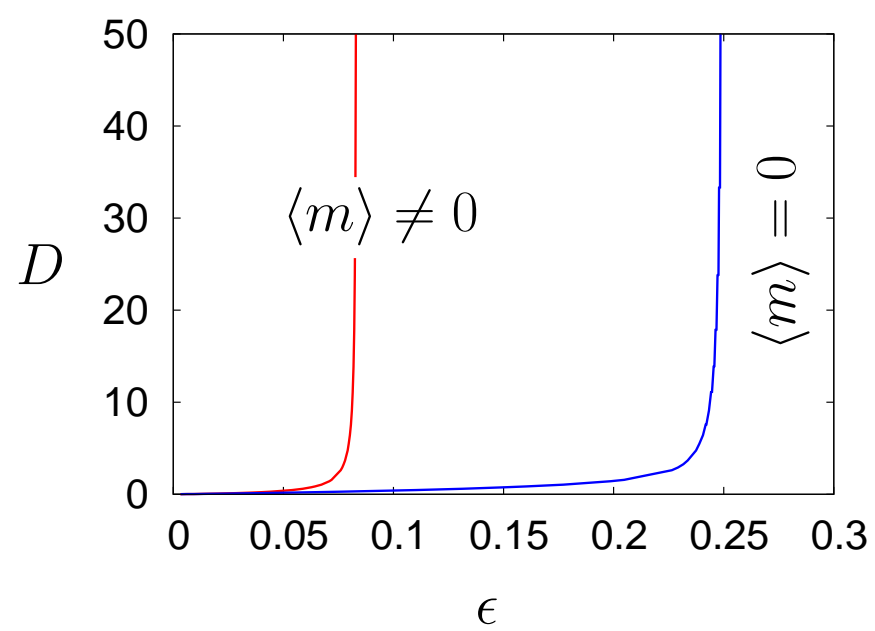

Figure 1. Dynamical phase diagram of our model in the $(\epsilon, D)$ plane for the choice of the waterbag initial state, Eq. (16). The line to the right is the thermodynamic phase boundary $D\left(\epsilon_{c}\right)$ between the magnetic and the non-magnetic phase, and is given by Eq. (13) with $\beta_{c}$ obtained by solving Eq. (12). The line to the left gives the dynamical phase boundary $D\left(\epsilon^{*}\right)$, and is given by Eq. (32).

We thus see that the frequency $\omega$ is real for $\epsilon>\epsilon^{*}$, given by

$$
\epsilon^{*}=\frac{D}{3+12 D},
$$

see Fig. (1). Therefore, unstable modes do not exist in this energy range so that the waterbag state (16) is linearly stable. Hence, QSS is observed. In this case, in a finite system, such a state eventually relaxes to Boltzmann-Gibbs equilibrium on a timescale over which non-linear correction terms should be added to the Vlasov equation [3].

On the other hand, for $\epsilon<\epsilon^{*}$, the waterbag state is unstable. Consequently, the perturbation $\delta f(\theta, \phi, t)$ grows exponentially fast towards Boltzmann-Gibbs equilibrium. Below $\epsilon^{*}$, on setting $\omega^{2}=-\Omega^{2}$ with real $\Omega$, we get $\delta f(\theta, \phi, t)=A e^{ \pm i \phi+\Omega t}$, where $A$ is a constant. Consequently, the average magnetization behaves as

$$
m(t) \sim \frac{1}{\sqrt{N}} e^{\Omega t} ; \quad \epsilon<\epsilon^{*},
$$

before it relaxes to the equilibrium value. From the above equation, it follows that for $\epsilon<\epsilon^{*}$, the relaxation timescale $\tau(N)$ over which the magnetization acquires the equilibrium value of $O(1)$ scales as $\ln N$. 

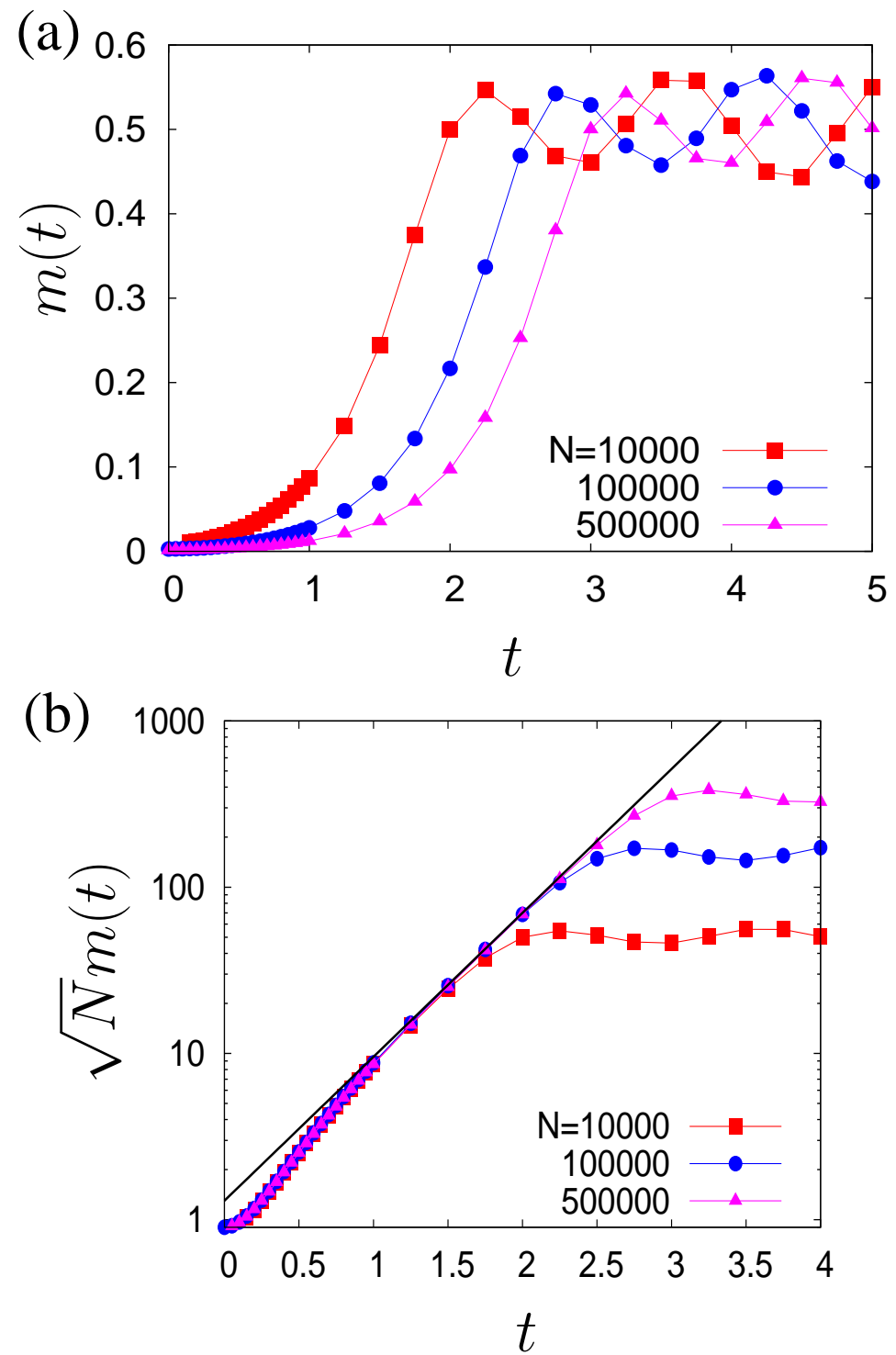

Figure 2. (a) Time evolution of average magnetization $m(t)$ in the unstable phase with energy density $\epsilon=0.0603$, the parameter $D=15$, and for systems of size $N=$ $10000,100000,500000$ (left to right). (b) Data collapse for the scaled magnetization $\sqrt{N} m(t)$ as a function of $t$, in accordance with Eq. (33). The black line represents the function $s(t) \sim e^{\Omega t}$, with $\Omega$ obtained from Eq. (31) by substituting $\omega^{2}=-\Omega^{2}$. Data averaging varies between $2 \times 10^{4}$ histories for the smallest system and 500 histories for the largest one.

\section{Numerical simulations}

In order to verify these features, we performed numerical simulations of the dynamics by integrating the equations of motion (44), (5) , (6) by using a fourth-order Runge Kutta method 


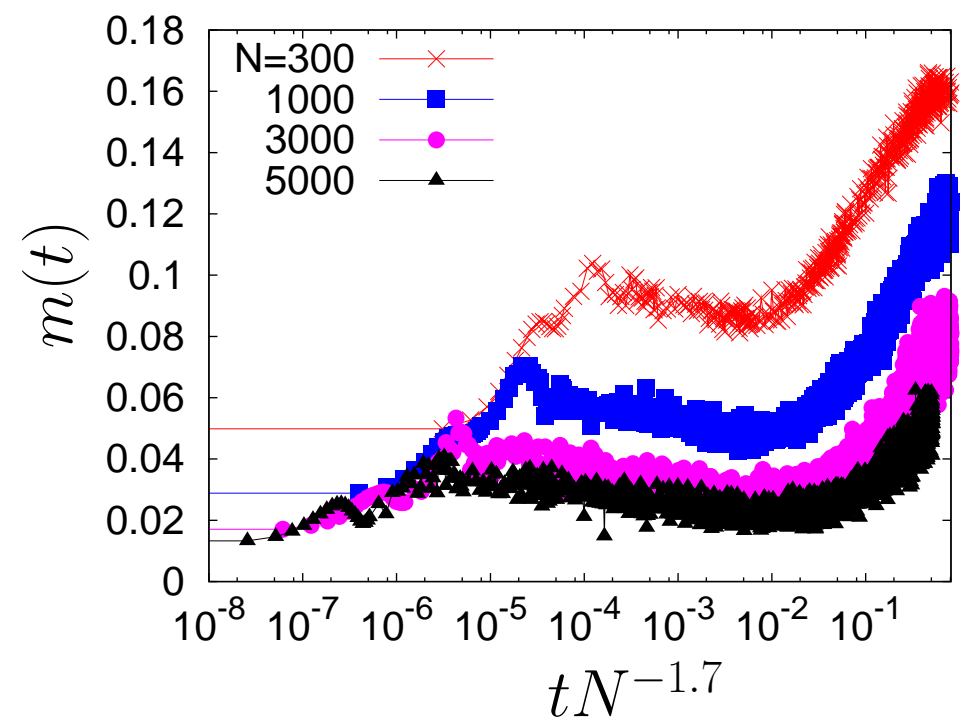

Figure 3. Average magnetization $m(t)$ as a function of $t N^{-1.7}$ in the stable phase with energy density $\epsilon=0.24$, the parameter $D=15$, and for systems of size $N=$ 300, 1000, 3000, 5000 (top to bottom). Data averaging varies between 300 histories for the smallest system and 25 histories for the largest one. The figure suggests a QSS life-time $\tau(N) \sim N^{1.7}$.

with time step equal to 0.01. For $\epsilon<\epsilon^{*}$, the results presented in Fig. 2(a) show that the magnetization grows fast towards equilibrium. On scaling the magnetization by $\sqrt{N}$, Fig. 2(b) shows a very good scaling collapse in accordance with the exponential growth predicted by Eq. (33). The growth rate $\Omega$ is in agreement with that obtained from Eq. (31) by substituting $\omega^{2}=-\Omega^{2}$. For energies $\epsilon^{*}<\epsilon<\epsilon_{c}$, when the waterbag state (16) is linearly stable, Fig. 3] suggests a much longer relaxation time $\tau(N) \sim N^{1.7}$. A similar scaling of the QSS relaxation time was also observed in the HMF model [18].

\section{Conclusions}

In conclusion, we addressed the ubiquity of non-Boltzmann quasistationary states (QSS) during relaxation of long-range systems. This was done by studying an anisotropic Heisenberg model of globally coupled classical spins evolving under classical spin dynamics. Quasistationary states have earlier been shown to occur in long-range interacting systems composed of particles (inertial rotors) which are evolving under particle dynamics dictated by the underlying Hamiltonian. Thus, our model provides a different possible setting for the occurrence of QSS under spin dynamics. By analyzing the Vlasov equation for the time evolution of the phase space distribution, we demonstrated that in this model, relaxation of 
a class of initial states in certain energy interval proceeds through intermediate QSS. These states have a lifetime that grows algebraically with the system size. This further establishes the possibility of long-range systems to exhibit quasistationarity under a broader class of dynamical processes.

\section{Acknowledgments}

We thank A. Bar, O. Cohen, T. Dauxois, O. Hirschberg, S. Levit and S. Ruffo for helpful discussions and comments on the manuscript. The support of the Israel Science Foundation

(ISF) and the Minerva Foundation with funding from the Federal German Ministry for Education and Research is gratefully acknowledged.

\section{References}

[1] Dauxois T, Ruffo S, Arimondo E and Wilkens M (ed) 2002 Dynamics and Thermodynamics of Systems with Long-Range Interactions (Lecture Notes in Physics vol. 602) (Springer-Verlag, Berlin).

[2] Campa A, Giansanti A, Morigi G and Sylos Labini F (ed) 2008 Dynamics and Thermodynamics of Systems with Long-Range Interactions: Theory and Experiments (AIP Conference Proceedings vol. 970) (Melville, New York).

[3] Campa A, Dauxois T and Ruffo S 2009 Phys. Rep. 48057.

[4] Dauxois T, Ruffo S and Cugliandolo L F (ed) 2010 Long-Range Interacting Systems (Oxford University Press, New York).

[5] Bouchet F, Gupta S and Mukamel D 2010 Physica A 3894389.

[6] Mukamel D 2010 Long-Range Interacting Systems ed T Dauxois, S Ruffo and L F Cugliandolo (Oxford University Press, New York) 33.

[7] Nicholson D R 1992 Introduction to Plasma Physics (Krieger Publishing Company, Florida).

[8] Landau L D and Lifshitz E M 1960 Electrodynamics of Continuous Media (Pergamon, London).

[9] Padmanabhan T 1990 Phys. Rep. 188285.

[10] Chavanis P H 2002 Dynamics and Thermodynamics of Systems with Long-range Interactions (Lecture Notes in Physics vol. 602) ed T Dauxois, S Ruffo, E Arimondo and M Wilkens (Springer-Verlag, Berlin).

[11] Lynden-Bell D and Wood R 1968 Mon. Not. R. Astron. Soc. 138495.

[12] Thirring W 1970 Z. Phys. 235339.

[13] Barré J, Mukamel D and Ruffo S 2001 Phys. Rev. Lett. 87030601.

[14] Mukamel D, Ruffo S and Schreiber N 2005 Phys. Rev. Lett. 95240604.

[15] Bouchet F and Barré J 2005 J. Stat. Phys. 1181073.

[16] Bouchet F, Dauxois T, Mukamel D and Ruffo S 2008 Phys. Rev. E 77011125.

[17] Antoni M and Ruffo S 1995 Phys. Rev. E 522361.

[18] Yamaguchi Y Y, Barré J, Bouchet F, Dauxois T and Ruffo S 2004 Physica A 33736.

[19] Campa A, Giansanti A and Morelli G 2007 Phys. Rev. E 76041117.

[20] Joyce M and Worrakitpoonpon T 2010 J. Stat. Mech.: Theory Exp. P10012.

[21] Teles T N, Levin Y, Pakter R and Rizzato F B 2010 J. Stat. Mech.: Theory Exp. P05007.

[22] Gabrielli A, Joyce M and Marcos B 2010 Phys. Rev. Lett. 105 210602. '

[23] Jain K, Bouchet F and Mukamel D 2007 J. Stat. Mech.: Theory Exp. P11008.

[24] Latora V, Rapisarda A and Ruffo S 1999 Phys. Rev. Lett. 832104. 
[25] Latora V, Rapisarda A and Tsallis C 2001 Phys. Rev. E 64056134.

[26] Bouchet F and Dauxois T 2005 Phys. Rev. E 72 045103(R).

[27] Nobre F D and Tsallis C 2003 Phys. Rev. E 68036115.

[28] Baldovin F and Orlandini E 2006 Phys. Rev. Lett. 96240602.

[29] Baldovin F and Orlandini E 2006 Phys. Rev. Lett. 97100601.

[30] Baldovin F, Chavanis P H and Orlandini E 2009 Phys. Rev. E 79011102.

[31] Gupta S and Mukamel D 2010 Phys. Rev. Lett. 105040602.

[32] Gupta S and Mukamel D 2010 J. Stat. Mech.: Theory Exp. P08026.

[33] Chavanis P H, Baldovin F and Orlandini E 2010 arXiv:1009.5603.

[34] Kac M, Uhlenbeck G E and Hemmer P C 1963 J. Math. Phys. 4216.

[35] Mermin N D 1967 J. Math. Phys. 81061.

[36] Barre J, Mukamel D and Ruffo S 2002 Dynamics and Thermodynamics of Systems with Long-range Interactions (Lecture Notes in Physics vol. 602) ed T Dauxois, S Ruffo, E Arimondo and M Wilkens (Springer-Verlag, Berlin).

[37] Braun W and Hepp K 1977 Comm. Math. Phys. 56101. 